\title{
Faune du sol et microflore. I. Modèle animal et paradoxe fonctionnel
}

\author{
M Pussard \\ INRA, station de recherches sur la faune du sol, 17, rue Sully, 21034 Dijon Cedex, France
}

(Reçu le 16 mai 1990; accepté le 6 mars 1991)

\begin{abstract}
Résumé - La lecture de la littérature nous apprend que la faune contribue à accroître de façon significative l'activité biologique d'un sol alors qu'elle ne participe que très peu au métabolisme global du milieu. Ce paradoxe caractérise le rôle des animaux dans le système des décomposeurs. En général, cet effet de stimulation est attribué à la libération par les animaux de substrats nutritifs supplémentaires utilisables par la microflore.

Cette interprétation n'est cependant pas compatible avec les caractéristiques biologiques du modèle animal : en raison de leur forte taille individuelle et de la biomasse de leurs populations, les animaux ont une activité métabolique globalement négligeable par rapport à celle de la microflore. Le niveau de stimulation observé en leur présence ne peut donc être attribué totalement à de banales relations trophiques. II est nécessaire d'envisager un autre mécanisme consommant moins d'énergie et de matières.

La comparaison des principales caractéristiques des divers groupes zoologiques montre que les protozoaires devraient jouer un rôle particulièrement important dans le système saprophage et en particulier dans l'agrosystème.
\end{abstract}

faune du sol / relation animal-microflore / stimulation microbienne / activité animale / métabolisme animal

Summary - Soil animals and microflora. I. Animal pattern and functional paradox. A survey of the literature shows that the direct contribution of the fauna to total soil metabolism is negligible but that the fauna increases soil activity significantly. This stimulating effect is generally attributed to animal activity which releases further nutriments for microflora.

This interpretation does not correspond with the biological characteristics of the animal model, due to their large size, and biomass of the animals, their metabolism is much lower than that of microflora. Animals cannot release sufficient nutriments to stimulate the microflora. Another stimulating mechanism which consumes less energy and matter therefore has to be considered. A survey of the animal pattern shows that protozoa must play an important role in decomposing systems and agrosystems.

soil fauna / animal-microflora relation / microbial stimulation / animal activity / animal metabolism

\section{INTRODUCTION}

En dépit du développement des techniques de culture hors sol, le sol demeure l'élément fondamental de la production végétale. Si l'efficacité des pratiques culturales de l'agriculture moderne a pu donner l'impression qu'il n'était pas nécessaire de mieux connaître les mécanismes biologiques de la fertilité des sols, l'accumulation des problèmes incite actuellement à revenir à une conception plus nuancée. En ce qui concerne les cultures hors sol, il n'est pas sans intérêt de signaler le fait que les dispositifs utilisés sont très rapidement colonisés par une microflore et une microfaune complexes : il est donc facile de prévoir que les facteurs biotiques ont aussi leur importance dans ce mode d'exploitation. En fait, c'est dans les milieux tels que prairies et forêts, moins perturbés par l'activité humaine, qu'il est possible d'étudier les processus biologiques naturels complexes qui fournissent à la végétation les nutriments nécessaires. Parmi ces processus, les relations faunemicroflore sont encore très imparfaitement connues.

Dans ce travail, l'objectif que l'on s'est fixé est de préciser les principaux modes d'action de la faune sur l'activité microbienne et cela sans se perdre dans l'infinie diversité des situations locales liée à la nature du sol, au climat, à la végétation, aux pratiques culturales, aux chaînes trophiques considérées, etc. 
Une abondante littérature a été consacrée au rôle de la faune dans le sol (en particulier Petersen et Luxton, 1982; Coleman, 1985; Anderson et al, 1985; Lebrun, 1987; Lavelle, 1988; Anderson, 1988; Persson, 1989). Dans l'ensemble, le rôle attribué aux animaux est paradoxal. En effet de très nombreux auteurs, travaillant dans des conditions différentes, ont conclu à un très net effet de stimulation de tous les processus de dégradation de la matière organique en présence d'animaux. À titre d'exemple, nous évoquerons ici 2 publications très souvent citées bien que leurs conclusions soient quelque peu contestées. Kurcheva (1960), puis Edwards et Heath (1963) ont montré qu'en présence d'animaux, la décomposition des feuilles de chêne est $3-5$ fois plus rapide qu'en présence de la seule microflore. Ce phénomène de stimulation lié à la faune est tout à fait général : il se manifeste aussi en milieu aquatique et en microcosmes simplifiés où les résultats sont moins contestables (Barsdate et al, 1974; Fenchel et Harrison, 1975). Le paradoxe réside dans le fait que l'intensité de la stimulation observée ne semble pas être en rapport avec le faible niveau des activités animales, niveau qui ressort des 2 constatations suivantes :

- les animaux ne représentent que $20 \%$ de la biomasse des organismes vivant dans le sol, racines exceptées (Bessard, 1961);

- la majorité des zoologistes s'accorde pour admettre que $10 \%$ au plus du métabolisme global du sol sont imputables à la faune (MacFadyen, 1961; Edwards et al, 1970; Syers et al, 1979; Persson et al, 1980; Satchell, 1983) d'où il pourrait être déduit que le rôle des animaux est insignifiant.

II existe donc bien un décalage considérable entre les conséquences fonctionnelles de la présence des animaux et le potentiel métabolique de ces derniers. Ainsi apparaît le caractère paradoxal du rôle des animaux dans le système des décomposeurs. Dès 1961, MacFadyen commentait ce phénomène de la manière suivante : It is only recently that we have begun to realize that animals may have effects on the metabolism of the soil out of proportion to their own respiration rates.

Pour expliquer l'augmentation de l'activité biologique en présence d'animaux 2 possibilités sont à envisager : ou bien les animaux agissent directement sur la matière organique, ou bien ils interviennent indirectement en modifiant l'activité de la microflore. Si la majorité des auteurs se rallie à la deuxième hypothèse, c'est toujours en évoquant la fourniture par les animaux de substrats supplémentaires et/ou de nutriments limitants $(N, P)$ à la microflore. De ce point de vue, les activités animales peuvent être réparties en activités mécaniques (fragmentation, brassage, fouissement, dissémination de germes) et en activités biochimiques (recyclage d'une partie de la biomasse consommée, excrétions, sécrétions). Cette théorie, relativement séduisante, n'a toutefois jamais été réellement démontrée.

Dans ce premier article, nous chercherons à savoir si les caractéristiques biologiques du modèle animal sont compatibles avec la quantité d'énergie que les animaux devraient dépenser pour fournir les substrats indispensables à la stimulation de la microflore. Dans un deuxième article, nous examinerons l'état des connaissances sur le régime alimentaire des animaux saprophages : en consommant de la biomasse microbienne ceux-ci pourraient contrôler le niveau des populations et stimuler ces dernières par un effet dépendant de la densité. Cette revue nous conduira à proposer une autre hypothèse pour expliquer de façon plus satisfaisante le paradoxe de l'action animale sur l'activité microbienne.

Malgré leur extrême diversité morphologique, biologique et fonctionnelle, les animaux du sol possèdent en commun des caractères essentiels qui les opposent aux bactéries et aux champignons : forte taille individuelle, mobilité, dépendance nutritionnelle vis-à-vis de la microflore, phagotrophie, longueur du cycle biologique.

Ci-dessous nous examinerons plus particulièrement les 4 premiers caractères afin de pouvoir mieux comparer l'activité animale à l'activité microbienne.

Il importe de préciser enfin que l'étude des relations faune-microflore ne saurait être limitée au seul milieu édaphique où l'acquisition des connaissances est ralentie par des difficultés méthodologiques évidentes. II est donc indispensables d'utiliser les progrès substantiels réalisés dans l'étude des animaux aquatiques dont les rapports avec la microflore posent des problèmes identiques.

\section{Taille individuelle, activité métabolique, biomasse et production}

\section{Taille individuelle et activité métabolique}

La grande diversité de taille individuelle, donc de poids individuel des animaux est une caractéristi- 
que majeure des groupes zoologiques représentés dans le sol. Cela ne doit cependant pas masquer le fait que les animaux, même les plus petits, ont une taille individuelle très supérieure à celle des microorganismes. Les animaux les moins volumineux sont sans conteste les protozoaires qui ne mesurent pas plus de quelques dizaines de $\mu \mathrm{m}$; les plus gros invertébrés sont les lombriciens qui atteignent une longueur de plusieurs dm. Le volume corporel d'un protozoaire de taille moyenne est d'environ 3000 fois plus grand que celui d'une bactérie et $10^{9}$ fois plus faible que celui d'un lombricien. De telles différences de volume et de poids individuel ont bien évidemment des répercussions importantes sur le potentiel métabolique relatif des organismes. C'est ainsi que l'activité métabolique ramenée à l'unité de poids ou taux d'activité $m$ (en kcal. $\mathrm{g}^{-1} \mathrm{j}^{-1}$ ) est liée au poids individuel ( $P$ en $\mathrm{g}$ ) par la relation empirique suivante :

$$
m=a P b
$$

où $b$ est un coefficient négatif dont la valeur absolue varie assez peu autour de 0,250 tandis que a dépend de l'activité considérée et du type d'organisme (Reichle, 1968; Fenchel, 1974; Blueweiss et al, 1978; Baldock et al, 1980; Lavigne, 1982). Ainsi le taux d'activité métabolique varie en raison inverse du poids individuel. Cette relation classique est très importante puisqu'elle permet de comparer les activités des populations d'organismes différents. En effet, si nous considérons 2 organismes de poids individuels très différents $\left(P_{1}>>P_{2}\right)$ dont les populations ont des biomasses inégales $\left(B_{1}<B_{2}\right)$ comme c'est le cas très souvent lorsqu'on compare un animal à une bactérie, le rapport des activités globales $\left(M_{1}\right.$, $\mathrm{M}_{2}$ ) des 2 populations est

$$
M_{1} / M_{2}=K\left(P_{2} / P_{1}\right)^{0,250} B_{1} / B_{2}
$$

Il en résulte à l'évidence que $M_{1}$ est beaucoup plus faible que $\mathrm{M}_{2}$ et que l'activité de la population animale $\left(M_{1}\right)$ est dans la plupart des cas si faible qu'elle peut être négligée par rapport à celle de la population microbienne $\left(\mathrm{M}_{2}\right)$. Cette constatation est très importante. À titre d'exemple nous pouvons prendre le cas des cultures mixtes de protozoaires et de bactéries : l'application des relations précédentes permet de montrer que la respiration des protozoaires est négligeable par rapport à celle des bactéries et que l'augmentation du dégagement de $\mathrm{CO}_{2}$ par rapport à la culture pure de bactéries provient non pas de la respiration des protozoaires mais de celles des bactéries (Coûteaux et al, 1988). De même, Baath et al (1981) sont conduits à admettre que la participation des nématodes à la respiration d'une culture mixte avec bactéries est tout à fait insignificante. À l'inverse, ces considérations montrent les risques d'erreur encourus lors de mesures respirométriques effectuées sur des animaux non débarassés de la microflore associée.

\section{Biomasse}

Dans le sol, les divers groupes animaux représentent des biomasses très différentes. C'est ainsi que le poids de lombriciens est d'environ 1t/ha (poids de matières fraîches) alors que le poids de protozoaires ne représente qu'une centaine de $\mathrm{kg}$ et celui de chaque groupe d'arthropodes s'exprime en $\mathrm{kg}$. Ces différences ont pu inspirer l'idée d'une hiérarchie fonctionnelle entre groupes zoologiques d'après la biomasse (cf par exemple Griffiths, 1990). En réalité la relation indiquée ci-dessus montre qu'à biomasse égale les fonctions des populations ne sont pas forcément égales mais varient en raison inverse du poids individuel moyen. La comparaison des productions des différents groupes d'organismes en fournit un bon exemple.

\section{Production}

La production annuelle de matière organique est une des manifestations de l'activité métabolique d'une population. Il est intéressant de l'exprimer sous forme du taux de renouvellement annuel ou taux de production (quotient de la production annuelle par la biomasse moyenne). En climat tempéré humide, le taux de production d'amibes ou de flagellés oscillerait, suivant les espèces, entre 50 et 300 (Stout et Heal, 1967); celui des thécamoebiens entre 14 et 25 (Lousier, 1974). En revanche, dans les pelouses sèches du Colorado la production annuelle des protozoaires ne représenterait que 3-4 fois la biomasse moyenne (Elliott et Coleman, 1977). Dans l'ensemble, les protozoaires ressemblent aux autres microorganismes par l'importance de leur production.

À l'opposé, les lombriciens ont un taux de production faible : en climat tempéré, Lumbricus terrestris (épianécique) et Allolobophora caliginosa (endogé) ont des taux de production respectifs de 0,45 (Lakhani et Satchell, 1970) et de 1,12,2 (Andersen, 1983). En climat tropical, les taux de production d'espèces endogées (Genres Mill- 
sonia et Dichogaster) oscillent entre 1,1 et 2,1 (Lamotte et Meyer, 1978). De fait, MacNeill et Lawton (1970) placent les lombriciens parmi les invertébrés à vie longue et à production faible. En effet, taux de renouvellement et durée de vie sont grosso modo inversement proportionnels (Lamotte et Meyer, 1978). Sur un vaste échantillonnage d'invertébrés, le taux de production oscille entre 0,13 et 6,3 (Lévèque et al, 1977).

Ces estimations de la production illustrent bien la relation existant entre activités métaboliques et poids individuel. En effet, si nous admettons que les protozoaires ont une biomasse 10 fois plus faible que celle des lombriciens et un taux de production 20 fois plus élevé, leur production annuelle serait le double de celle des lombriciens. Bien plus, l'application des relations proposées par Fenchel (1974) permet de calculer que, toujours dans l'hypothèse d'un rapport de biomasse de 1 à 10, les protozoaires auraient une production 10 fois plus élevée que celle des lombriciens. Ainsi, chez ces derniers, l'importance de la biomasse est compensée par une activité métabolique relativement modeste.

En réalité, sur le plan fonctionnel, la production est un paramètre beaucoup plus significatif que la biomasse : alors que cette dernière correspond à la quantité de nutriments immobilisés à un instant donné par les organismes, le taux de production traduit l'intensité du renouvellement de ces nutriments, donc leur mobilité et leur degré de disponibilité pour la communauté.

En conclusion, ces considérations sur la taille individuelle des animaux sont en parfait accord avec le constat d'une faible participation des animaux au métabolisme global du sol. En effet, la disproportion évoquée entre activités des lombriciens et des protozoaires s'accentue considérablement lorsque la comparaison porte non plus sur les protozoaires mais sur les bactéries. En conséquence le flux d'énergie et de matière passant par les animaux doit être considéré comme relativement peu important.

Les grandes différences de poids individuel ont enfin un lien évident avec le degré de dispersion de la biomasse des divers groupes zoologiques dans le sol. Ainsi, en dépit de leur forte biomasse, les lombriciens ne sont que quelques centaines d'individus au $\mathrm{m}^{2}$, tandis que sur cette même surface Stout et Heal (1967) estiment le nombre de protozoaires à $1,7 \times 10^{12}$. Alors que l'activité des lombriciens est très localisée, celle des protozoaires est extrêmement diffuse.

\section{Mobilité et activités mécaniques}

Une particularité essentielle du modèle animal est la mobilité qui confère à l'individu un certain espace vital et la possibilité d'une optimisation permanente des conditions de vie. Cette faculté est primordiale en milieu édaphique puisque la matrice solide ne permet pas un renouvellement suffisant des phases fluides, renouvellement indispensable aux animaux fixés, vivant en milieu aquatique.

La mobilité est à l'origine d'un certain nombre d'effets des animaux sur la microflore (brassage, fouissement, dissémination de germes) souvent invoqués pour expliquer la stimulation de la microflore. Mais, elle implique des dépenses énergétiques : ses divers effets vont donc être limités par l'énergie dont disposent les animaux.

Du point de vue de l'espace vital, les animaux du sol sont plus ou moins indépendants de la porosité du sol suivant qu'ils sont ou non fouisseurs.

De très nombreuses espèces doivent se contenter de coloniser le réseau lacunaire préexistant : taille et rigidité du corps sont alors des facteurs qui limitent l'espace vital.

Chez les espèces fouisseuses (certains arthropodes, de nombreux lombriciens), la contrainte de taille est moins grande mais l'espace vital est acquis au prix d'une dépense énergétique. Chez les lombriciens, le fouissement implique essentiellement le tassement du sol (Lee; 1985) : la lumière de la galerie est donc formée aux dépens de la porosité du sol; il en résulte que la paroi des galeries est constituée de terre tassée, facteur qui peut contribuer à diminuer l'infiltration latérale de l'eau et la diffusion gazeuse. Les pressions exercées par les lombriciens lors du fouissement sont nettement inférieures à celles mesurées au niveau des racines (MacKenzie et Dexter, 1988). Dans un sol compact, le fouissement est assuré par ingestion de terre. Néanmoins, il s'est parfois révélé difficile d'introduire une population lombricienne dans des sols très tassés (Rushton, 1986). 
Il faut signaler enfin que les galeries de lombriciens ne représentent qu'une faible fraction de la porosité totale du sol (moins de 1\% selon LopesAssad, 1987).

\section{Dépendance nutritionnelle Besoins en facteurs de croissance et emprunts enzymatiques}

Le modèle animal est caractérisé par une régression du pouvoir de synthèse, très marquée déjà au niveau des protozoaires (Lwoff, 1943). Cette régression physiologique a 2 sortes de conséquences sur le comportement alimentaire des animaux.

En premier lieu, ceux-ci doivent emprunter à d'autres organismes les molécules ou précurseurs dont ils ne savent pas faire la synthèse (facteurs de croissance ou vitamines). Ces substances indispensables proviennent des proies vivantes chez les espèces carnassières ou phytophages et de la microflore chez les espèces saprophages qui consomment de la matière organique en décomposition.

En second lieu, puisque les animaux sont censés ne disposer que d'une gamme réduite d'enzymes digestifs propres, il faut bien admettre qu'ils ne peuvent digérer que des substances organiques simples (Lavelle, 1988) ou les constituants du protoplasme (Nielsen, 1967) à l'exclusion des polymères structuraux (Zinkler, 1983) qui représentent pourtant la fraction essentielle de la matière organique parvenant au sol. Pour profiter de ces ressources nutritives récalcitrantes, les animaux saprophages doivent en principe utiliser l'activité enzymatique d'une microflore symbiotique ou en transit et même les enzymes libres présentes dans le milieu (Hassal et Jennings, 1975; Martin, 1984; Martin et Kukor, 1984).

Certains travaux (Nielsen, 1962; Zinkler, 1971; Luxton, 1972; Dash et al, 1981) tendent à faire croire que les animaux du sol sont capables, par eux-mêmes, de digérer les polysaccharides structuraux des végétaux et qu'ils peuvent de ce fait être considérés comme des décomposeurs primaires. En fait, les démonstrations expérimentales ne sont pas probantes puisque les auteurs n'ont pris aucune précaution pour éliminer la microflore d'accompagnement et ses enzymes; il en est de même dans la controverse relative à l'existence de cellulases animales. II est très regrettable que les zoologistes du sol confondent si facilement animal et microflore associée.
Il est néanmoins clair que la régression physiologique a placé les animaux saprophages sous une double dépendance vis-à-vis de la microflore : dépendance vitaminique et dépendance enzymatique.

\section{Phagotrophie. Choix alimentaire, défécation et excrétion}

\section{Phagotrophie}

Le mode d'alimentation est avec la mobilité, le caractère distinctif le plus spectaculaire du modèle animal : au lieu d'avoir une digestion extracorporelle comme les bactéries et les champignons, les animaux ingèrent les aliments et les digèrents à l'intérieur de leur corps. Ce mode d'alimentation, la phagotrophie, procure de nombreux avantages : économie en moyens enzymatiques, meilleur contrôle des activités enzymatiques, possibilité de favoriser sélectivement certains groupes microbiens, récupération plus complète des produits de la digestion. II convient de signaler que les protozoaires phagotrophes occupent une place intermédiaire entre modèle animal et modèle microbien puisqu'ils sont capables d'émettre des hydrolases extracellulaires et d'absorber les petites molécules à travers leur surface cellulaire (Tiedtke et Rasmussen, 1988).

La phagotrophie correspond à l'existence d'un appareil digestif plus ou moins complexe. $\mathrm{Ce}$ compartiment corporel a pour fonction de séquestrer, de conditionner et de digérer une fraction de milieu ambiant, puis d'assimiler les produits de la digestion et enfin de rejeter les éléments non digérés. Comme nous le préciserons ultérieurement, les microorganismes ingérés ne se heurtent pas, dans l'appareil digestif, aux défenses spécifiques mises en œuvre pour empêcher leur pénétration dans les autres compartiments de l'organisme : l'exemple classique à cet égard est celui des lombriciens (Lassegues, 1986; Lassalle et al, 1988).

L'ingestion des aliments est directement liée au flux énergétique : il n'est donc pas étonnant que chez les arthropodes la quantité d'aliments ingérée par unité de poids suive en principe la relation générale indiquée au chapitre 1 (Reichle, 1968). Chez les espèces géophages toutefois, les quantités ingérées sont relativement importantes puisqu'elles comportent une fraction minérale considérable : elles sont d'autant plus grandes que le pourcentage de matière organique est plus faible (Lavelle et al, 1983). 


\section{Choix alimentaire}

L'ingestion ne se fait pas au hasard : elle obéit à un choix mis en évidence chez les protozoaires, les arthropodes et les lombriciens. Dans ce dernier cas, c'est à un tel choix que doivent être attribuées certaines différences de composition entre turricules récoltés sur le terrain et échantillons du sol correspondant : les turricules sont plus riches non seulement en éléments $(N, P, K)$ assimilables mais également en éléments totauX et même en matières organiques (Lunt et Jacobson, 1944). Ces particularités de composition disparaissent dès lors que les lombriciens sont expérimentalement placés dans des conditions où le choix devient difficile (Watanabe, 1975) : les turricules sont alors plus pauvres en matières organiques (pertes évaluées à 9-19\% par Barois et Lavelle, 1986). En raison de l'existence d'un tel choix, il est difficile d'admettre avec Bouché et al (1983) que le contenu du tube digestif des lombriciens puisse constituer un échantillon représentatif de sol.

La phagotrophie a 2 conséquences importantes : la défécation et l'excrétion.

\section{La défécation}

Ce phénomène revêt une importance particulière chez les animaux saprophages qui ne digèrent qu'une faible partie du bol alimentaire. Ils rejettent la fraction non digérée sous forme de pelotes fécales, fèces ou turricules. Ces éléments dont la composition dépend du choix alimentaire contiennent les substances «récalcitrantes» mélangées à des substances émises par l'animal. Après leur rejet dans le milieu extérieur, ils sont le siège d'une prolifération microbienne dont le résultat est une dégradation plus poussée des substances organiques récalcitrantes, apparition de molécules assimilables et augmentation transitoire de la biomasse microbienne; cette évolution justifie souvent leur ingestion (coprophagie) par des individus de la même espèce ou d'espèces différentes. La succession de tels cycles d'ingestion-défécation qui aboutit à la minéralisation progressive des polymères (Hargrave, 1976) suppose l'interaction étroite de l'animal et de la microflore.

\section{Excrétion}

Nous avons indiqué précédemment que les animaux étaient censés n'assimiler que des substances provenant de la digestion du proto- plasme. La fraction assimilée par eux a donc des rapports $\mathrm{C} / \mathrm{N}$ et $\mathrm{C} / \mathrm{P}$ très proches des rapports corporels de l'animal lui-même. Pour assurer une relative stabilité de sa composition, ce dernier doit donc, pour compenser les pertes en carbone liées à la respiration, rejeter azote et phosphore en excès. Cette excrétion se fait sous forme directement assimilable par les plantes $\left(\mathrm{N}-\mathrm{NH}_{4}\right.$ et phosphates) ou sous une forme très vite minéralisée par la microflore (urée, acide urique, allentoïne, guanine). Ce phénomène d'excrétion est général sauf chez certains groupes qui ont développé des mécanismes de stockage tels les termites pour l'azote. Sur le plan fonctionnel, l'excrétion animale est un processus de minéralisation de la matière organique. Par leur minéralisation permanente, les animaux s'opposeraient au modèle bactérien qui, dans les conditions normales, immobiliserait azote et phosphore alors que le modèle fongique, comme le modèle animal, aurait plutôt tendance à minéraliser ces éléments (Wright et Coleman, 1988).

L'excrétion correspond à une libération de nutriments utilisables par la microflore : d'après les considérations exposées dans le paragraphe 1 , l'importance de ce phénomène ne devrait pas être surestimée. S'il est tout à fait vraisemblable que l'azote puisse passer directement des lombriciens aux plantes (Bouché et Ferrière, 1986) it est douteux que cela puisse représenter plus de $80 \%$ des besoins des plantes. En prairie, Syers et al (1979) considèrent comme négligeable la contribution des lombriciens à la minéralisation de l'azote tandis que dans les litières forestières Anderson et Ineson (1984) estiment à 7,5\% seulement le taux d'azote minéralisé d'origine animale; il convient de remarquer que cette dernière estimation est plus probable puisqu'elle est en parfait accord avec le pourcentage de l'activité métabolique du sol imputable aux animaux.

Les chercheurs du Natural Resource Ecology Laboratory de Fort Collins attribuent aussi le rôle joué par les protozoaires dans la minéralisation de l'azote à l'excrétion par eux de $\mathrm{N}-\mathrm{NH}_{4}$ et vont même jusqu'à supposer que ce phénomène puisse couvrir une part importante des besoins en azote des végétaux (Anderson et al, 1981; Woods et al, 1982; Clarholm, 1985). En critiquant cette interprétation, Coûteaux et al (1988) remarquent que l'excrétion d'azote ammoniacal n'a vraiment été démontrée que chez des protozoaires maintenus en culture axénique. De fait, dans des cultures monoxéniques du cilié Colpoda aspera, Palka (1988) n'a pu déceler aucune excrétion de cette forme d'azote. En réalité, dans 
cette expérience, compte tenu de la relative faiblesse de leur biomasse, les ciliés pourraient n'avoir pas excrété assez d'azote pour dépasser le seuil de détection de la méthode de dosage utilisée. D'après les données publiées par Dolphin (1976) dans le cas de l'amibe Acanthamoeba castellanii entretenue en culture axénique, 1 ppm de $\mathrm{N}-\mathrm{NH}_{4}$ est rejeté par 15000 amibes en $7 \mathrm{j}$. II s'avère ainsi que l'excrétion azotée des protozoaires, tout comme le rejet de $\mathrm{C}-\mathrm{CO}_{2}$, puisse être très faible et négligeable par rapport à celle des bactéries.

Puisque les bactéries accumulent le phosphore sous forme de polyphosphates, les protozoaires bactériophages pourraient jouer un rôle dans la minéralisation de cet élément. En milieu aquatique, Johannes (1965) considère que l'excrétion de phosphates par les protozoaires est le processus le plus important tandis que Barsdate et al (1974) envisagent plutôt une libération accrue de phosphates par les bactéries elles-mêmes en présence de protozoaires. Dans le sol, Cole et al (1978) sont plutôt favorables à l'interprétation de Johannes. II semble cependant que la thèse de Barsdate et al (1974) soit plus conforme aux caractéristiques du modèle animal.

En conclusion, les quantités d'azote et de phosphore excrétées par les animaux sont vraisemblablement très faibles et ne peuvent être en rapport avec l'augmentation de la minéralisation de la matière organique constatée en présence des animaux.

\section{Conclusions}

Nous devons d'abord reconnaître que les principaux caractères du modèle animal sont en accord avec le principe reconnu d'une faible participation animale au métabolisme global du sol. Dans ces conditions, est-il possible d'expliquer l'importance paradoxale de la stimulation de la minéralisation en présence d'animaux?

Pour répondre à cette question nous prendrons comme exemple celui mentionné dans l'introduction et relatif à la dégradation des feuilles de chêne qui serait 3-5 fois plus rapide en présence d'animaux qu'en leur absence. En premier lieu, les animaux ne peuvent pas dégrader eux-mêmes la matière organique, même à un rythme beaucoup plus faible, non seulement en raison de leur compétence enzymatique limitée mais surtout en raison de la faible intensité de leur métabolisme. Leur action ne peut donc s'exercer que par l'intermédiaire de la microflore libre ou commensale. Nous avons signalé que c'est précisément l'hypothèse à laquelle se rallient tous les auteurs en supposant l'intervention de banales relations trophiques : les animaux rejetteraient des nutriments (sécrétions, excrétions) et/ou démasqueraient des substances (fragmentation, brassage, dissémination) inaccessibles autrement à la microflore. Nutriments et substrats devraient toutefois apparaître à un rythme tel que la microflore puisse multiplier son activité par 3 ou 5 , ce qui, compte tenu du rendement d'assimilation de la microflore, supposerait une activité animale particulièrement intense, activité elle-même soutenue par l'utilisation préalable de produits microbiens, source trophique apparemment essentielle aux animaux saprophages (Pussard, 1991). Ceci conduirait à admettre que la faune puisse avoir dans ce scénario une activité métabolique globalement supérieure à celle de la microflore pour entretenir le niveau de stimulation signalé par certains auteurs.

Pour ces diverses raisons, il semble difficile d'accepter à la fois un tel niveau de stimulation et l'hypothèse d'un mécanisme reposant uniquement sur de banales relations trophiques. II faut préciser d'abord que des travaux plus récents (Baath et al, 1978; Setälä et al, 1988; Clarholm, 1989) concluent en fait à un niveau beaucoup plus modeste de la stimulation exercée par la faune. II est certes possible d'objecter aussi que les activités animales puissent avoir un impact disproportionné dans la mesure où les processus de dégradations microbiennes seraient ralentis soit par une très faible disponibilité en azote et/ou en phosphore, soit par des phénomènes d'antibiose. Dans les 2 cas l'irruption d'un animal restituant azote et phosphore prélevés ailleurs et bouleversant les rapports existant localement entre microorganismes pourrait suffire à relancer localement l'activité microbienne. II est évidemment difficile d'évaluer l'impact d'actions aussi localisées sur l'évolution globale de la minéralisation dans la litière. Néanmoins, il nous semble indispensable de suivre enfin le conseil que MacFadyen donnait dès 1961 aux zoologistes: We should take seriously the possibility that soil animals promote metabolism on a vastly greater scale than that which results from their own internal physiology et de rechercher une hypothèse qui mettrait en cause un mécanisme de nature catalytique n'exigeant des animaux qu'une dépense énergétique modeste. Dans la deuxième partie de cet article, nous envisagerons la généralisation d'un tel mécanisme 
mis en évidence récemment chez les protozoaires (Levrat, 1990).

Quels que soient les mécanismes en cause, les animaux, du fait de la faiblesse relative de leur densité de population, ont une action très localisée que tempèrent quelque peu leurs déplacements. Seuls les protozoaires, et à un moindre degré les plus petits invertébrés tels les nématodes, pourraient agir sur la microflöre de façon diffuse dans le sol. De plus, l'activité des protozoaires est liée à celle de la microflore bactérienne : protozoaires et bactéries ont en effet les mêmes exigences en eau; la reprise d'activité (dékystement) et la croissance de la plupart des protozoaires semblent dépendre de la croissance bactérienne; en raison de leur temps de génération court, les protozoaires sont pratiquement les seuls organismes bactériophages à se multiplier assez rapidement pour opposer une réponse numérique à la multiplication bactérienne. Enfin, les protozoaires seraient beaucoup moins perturbés que les invertébrés par les pratiques culturales (Hunt et al, 1989). D'où la nécessité d'accorder à ces animaux microscopiques une attention particulière.

Il pourrait paraître superflu d'avoir évoqué, comme nous l'avons fait, les caractères du modèle animal et des principes biologiques élémentaires. La nécessité de le faire est apparue à la lecture de la littérature consacrée au rôle de la faune dans le sol : nombreuses sont les hypothèses de travail et les interprétations de résultats expérimentaux qui sont contraires à de tels principes. À titre d'exemple, nous évoquerons à nouveau les théories qui attribuent une fraction importante de l'azote puisé par les plantes tantôt aux protozoaires, tantôt aux lombriciens : il n'est pas sans intérêt de remarquer que ces théories, trop exclusives, tendent à s'annuler réciproquement. Le rappel des caractères du modèle animal nous a permis de définir quelques notions générales permettant d'orienter le choix des hypothèses de travail et de juger les interprétations proposées. Certes la remise en cause de tels principes théoriques est toujours possible mais à la condition expresse d'une démonstration expérimentale particulièrement irréprochable.

\section{RÉFÉRENCES}

Andersen NC (1983) Nitrogen turnover by earthworms in arable plots treated with farmyard manure and slurry. In: Earthworm ecology from Darwin to vermiculture (JE Satchell, ed). Chapman \& Hall Londres, 139-150
Anderson JM (1988) The role of soil fauna in agricultural systems. In: Advance in nitrogen cycling in agricultural ecosystems (JR Wilson, ed) CAB int, 89112

Anderson JM, Ineson P (1984) Interactions between microorganisms and soil invertebrates in nutrient flux pathways of forest ecosystems. In: Invertebrate-microbial interactions (JM Anderson, ADM Rayner, DWH Walton, eds) Cambridge Univ Press, 59-88

Anderson RV, Coleman DC, Cole CV (1981) Effects of saprotrophic grazing on net mineralization. Ecol Bull 33, 201-216

Anderson JM, Huish SA, Ineson P, Leonard MA, Splatt PR (1985) Interactions of invertebrates, microorganisms and tree roots in nitrogen and mineral element fluxes in deciduous woodland soils. In: Ecological interactions in soil, plant, microbes and animals (AH Fitter, D Atkinson, DJ Read, MB Usher, eds), Blackwell Sci Publ, 377-392

Baath $E$, Lohm U, Lundgren B, Rosswall $T$, Söderström B, Sohlenius B, Wiren A (1978) The effect of nitrogen and carbon supply on the development of soil organism populations and pine seedlings : a microcosm experiment. Oikos 31, 153-163

Baath $E$, Lohm U, Lundgren B, Rosswall T, Söderström B, Sohlenius B (1981) Impact of microbialfeeding animals on total soil activity and nitrogen dynamics: a soil microcosm experiment. Oikos 37, 257-264

Baldock BM, Baker JH, Sleigh MA (1980) Laboratory growth rates of six species of freshwater Gymnamoeba. Oecologia 47, 156-159

Barois I, Lavelle P (1986) Changes in respiration rate and some physicochemical properties of a tropical soil during transit through Pontoscolex corethrurus (Glossoscolecidae, Oligochaeta). Soil Biol Biochem $18,539-541$

Barsdate RJ, Prentki RT, Fenchel T (1974) Phosphorus cycle of model ecosystems: significance for decomposer food chains and effect of bacterial grazers. Oikos 25, 239-251

Bessard A (1961) La faune du sol. Son rôle et son intérêt agronomique. Rev Zool Agric Appl, 1-31

Blueweiss L, Fox H, Kudzma V, Nakashima D, Peters R, Sams R (1978) Relationships between body size and some life history parameters. Oecologia 37 , 257-272

Bouché MB, Ferrière G (1986) Cinétique de l'assimilation de l'azote d'origine lombricienne par une végétation prairiale non perturbée. CR Séances Acad Sci Paris 302, 75-80

Bouché MB, Rafidison Z, Toutain F (1983) Étude de l'alimentation et du brassage pédo-intestinal du lombricien Nicodrilus velox (Annelida, Lumbricidae) par l'analyse élémentaire. Rev Ecol Biol Sol 20, 4975

Clarholm M (1985) Interactions of bacteria, protozoa and plants leading to mineralization of soil nitrogen. Soil Biol Biochem 17, 181-187 
Clarholm M (1989) Effects of plant-bacterial-amoebal interactions on plant uptake of nitrogen under field conditions. Biol Fertil Soils 8, 373-378

Cole CV, Elliott ET, Hunt HW, Coleman DC (1978) Trophic interactions in soils as they affect energy and nutrient dynamics. V. Phosphorus transformations. Microb Ecol 4, 381-387

Coleman DC (1985) Through a ped darkly: an ecological assessment of root-soil-microbiola-faunal interaction. In: Ecological interactions in soil (AH Fitter, D Atkinson, DJ Read, MB Usher, eds) Blackwell Sci Publ, 1-21

Coûteaux MM, Faurie G, Palka L, Steinberg C (1988) La relation prédateur-proie (protozoaires-bactéries) dans les sols : rôle dans la régulation des populations et conséquences sur les cycles du carbone et de l'azote. Rev Ecol Biol Sol 25, 1-31

Dash MC, Nanda B, Mishra PC (1981) Digestive enzymes in three species of Enchytraeidae (Oligochaeta). Oikos 36, 316-318

Dolphin WD (1976) Nitrogen metabolism in Acanthamoeba castellanii: amino acid consumption and composition patterns. Proc lowa Acad Sci 82, 171174

Edwards CA, Heath GW (1963) The role of soil animals in breakdown of leaf material. In: Soil Organisms ( $J$ Doeksen, J Van Der Drift, eds), North Holland Publ Co, Amsterdam, 76-85

Edwards CA, Reichle DE, Crossley DA Jr (1970) The role of soil invertebrates in turnover of organic matter and nutrients. In: Analysis of temperate forest ecosystems (DE Reichle, ed) Springer-Verlag, 147172

Elliott ET, Coleman DC (1977) Soil protozoan dynamics in a shortgrass prairie. Soil Biol Biochem 9, 113-118

Fenchel T (1974) Intrinsic rate of natural increase: the relationship with body size. Oecologia 14, 317-326

Fenchel T, Harrison P (1975) The significance of bacterial grazing and mineral cycling for the decomposition of particulate detritus. In: The role of terrestrial and aquatic organisms in decomposition processes (JM Anderson, A MacFadyen, eds) 285-299

Griffiths BS (1990) A comparison of microbial feeding nematodes and protozoa in the rhizosphere of different plants. Biol Fertil Soils 9, 83-88

Hargrave BT (1976) The central role of invertebrate faeces in sediment decomposition. In: The role of terrestrial and aquatic organisms in decomposition processes (JM Anderson, A MacFadyen, eds) Blackwell Sci Publ, 301-321

Hassall M, Jennings JB (1975) Adaptative features of gut structure and digestion physiology in the terrestrial isopod Philoscia muscorum (Scop.) Biol Bull 149, 348-364

Hunt HW, Elliott ET, Walter DE (1989) Inferring trophic interactions from pulse dynamics in detrital food webs. Plant Soil 115, 247-259

Johannes RE (1965) Influence of marine protozoa on nutrient regeneration. Limnologica 10, 434-442
Kurcheva GF (1960) Role of invertebrates in the decomposition of oak litter. Sov Soil Sci 4, 360-365

Lakhani KH, Satchell JE (1970) Production by Lumbricus terrestris (L). J Anim Ecol 39, 473-492

Lamotte M, Meyer JA (1978) Utilisation du taux de renouvellement $P / B$ dans l'analyse du fonctionnement énergétique des écosystèmes. $C R$ Séances Acad Sci Paris, 286D, 19, 1387-1390

Lassalle F, Lassegues M, Roch C (1988) Protein analysis of earthworm coelomic fluid. IV. Evidence, activity induction and purificaton of Eisenia fetida andrei lysozyme (Annelidae). Comp Biochem Physiol 91B, 187-192

Lassegues M (1986) Étude des activités antibactériennes humorales et cellulaires du lombricien Eisenia fetida andrei. Thèse, Bordeaux, $70 \mathrm{p}$

Lavelle P (1988) Earthworm activities and the soil system. Biol Fertil Soils 6, 237-251

Lavelle P, Zaidi Z, Schaefer R (1983) Interactions between earthworms, soil organic matter and microflora in an african savanna soil. In: New trends in soil biology ( $P$ Lebrun, HM André, A De Medts, C Gregoire-Wibo, G Wauthy, eds) Dieu-Brichart Ottignies-Louvain la Neuve, 253-259

Lavigne DM (1982) Similarity in energy budgets of animal populations. J Anim Ecol 51, 195-206

Lebrun P (1987) Quelques réflexions sur les rôles exercés par la faune édaphique. Rev Ecol Biol Sol 24, 495-502

Lee KE (1985) Earthworms. Their ecology and relationships with soils and land use. Acad Press, Australie, $411 p$

Lévèque C, Durand JR, Ecoutin JM (1977) Relations entre le rapport $P / B$ et la longévité des organismes. Cah ORSTOM (Hydrobiol) 11, 17-31

Levrat $P$ (1990) Contribution à l'étude des interactions entre protozoaires et microflore du sol. Effet d'une amibe bactériophage Acanthamoeba catellanii sur le métabolisme de Pseudomonas flurescents. Thèse, Lyon, $115 p$

Lopes-Assad ML (1987) Contribution à l'étude de la macroporosité lombricienne de différents types de sols de France. Thèse, Montpellier

Lousier JD (1974) Effects of experimental soil moisture fluctuations on turnover rates of Testacea. Soil Biol Biochem 6, 19-26

Lunt HA, Jacobson HGM (1944) The chemical composition of earthworm casts. Soil Sci 58, 367-375

Luxton M (1972) Studies on the oribatid mites of a Danish beech wood soil. Pedobiologia 12, 434-463

Lwoff A (1943) L'évolution physiologique. Étude des pertes de fonctions chez les microorganismes. Hermann, Paris, $308 p$

MacFadyen A (1961) Metabolism of soil invertebrates in relation to soil fertility. Ann App/ Biol 49, 215-218

MacKenzie BM, Dexter AR (1988) Radial pressures generated by the earthworm Aporrectodea rosea. Biol Fertil Soils 5, 328-332 
Macneill S, Lawton JH (1970) Annual production and respiration in animal populations. Nature (Lond) $225,472-474$

Martin MM (1984) The role of ingested enzymes in the digestive processes of insects. In: Invertebratemicrobial interactions (JM Anderson, ADM Rayner, DWH Walton, eds) Cambridge Univ Press, 155-172

Martin MM, Kukor JJ (1984) Role of mycophagy and bacteriophagy in invertebrate nutrition. In: Current perspectives in microbial ecology (MJ Klug, CA Reddy, eds), Am Soc Microbiol, Washington DC, 257-263

Nielsen CO (1962) Carbohydrases in soil and litter invertebrates. Oikos 13, 200-215

Nielsen CO (1967) Nematoda. In: Soil Biology (A Burges, F Raw, eds) Acad Press, Londres, New York, 197-211

Palka $L$ (1988) Rôle des protozoaires bactériophages du sol dans la minéralisation de l'azote en conditions gnotobiotiques. Thèse, Clermont-Ferrand, $114 p$

Persson T (1989) Role of soil animals in C and $\mathrm{N}$ mineralisation. Plant Soil 115, 241-245

Persson T, Baath E, Clarholm M, Lundkvist $H$, Söderström BE, Sohlenius B (1980) Trophic structure, biomass dynamics and carbon metabolism of soil animals in a Scots pine forest. Ecol Bull 32, 419459

Petersen H, Luxton M (1982) A comparative analysis of soil fauna populations and their role in decomposition processes. Oikos 39, 287-388

Pussard M (1991) Faune du sol et microflore II. Saprophagie, prédation et médiation chimique. Agronomie (accepté pour publication)

Reichle DE (1968) Relation of body size to food intake, oxygen consumption and trace element metabolism in forest floor arthropods. Ecology 49, 535542

Rushton SP (1986) The effects of soil compaction on Lumbricus terrestris and its possible implications for populations on land reclaimed from open-cast total mining. Pedobiologia 29, 85-90

Satchell JE (1983) Earthworm microbiology. In: Earthworm ecology from Darwin to vermiculture (JE Satchell, ed) Chapman and Hall, Londres, New York, 351-364

Setälä H, Haimi J, Huhta V (1988) A microcosm study of the respiration and weight loss in birch litter and raw humus as influenced by soil fauna. Biol Fertil Soils 5, 282-287

Stout JD, Heal OW (1967) Protozoa. In: Soil Biology (A Burges, E Raw, eds) Acad Press, Londres, New York, 149-195

Syers JK, Sharpley AN, Keenney DR (1979) Cycling of nitrogen by surface-casting earthworms in a pasture ecosystem. Soil Biol Biochem 11, 181-185

Tiedtke A, Rasmussen L (1988) Lysosomal enzymes in extracellular digestion in the unicellular eukaryote Tetrahymena. J Cell Physiol 136, 554-556

Watanabe $\mathrm{H}$ (1975) On the amount of cast production by the Megascolecid earthworm Pheretima hupeiensis. Pedobiologia 15, 20-28

Woods LE, Cole CV, Elliott ET, Anderson RV, Coleman DC (1982) Nitrogen transformations in soil as affected by bacterial-microfaunal interactions. Soil Biol Biochem 14, 93-98

Wright DH, Coleman DC (1988) Soil fauna vs. fertilization effects on plant nutrition: results of a biocide experiment. Biol Fertil Soils 7, 46-52

Zinkler D (1971) Carbohydrasen streubewohnender Collembolen und Oribatiden. In: Organismes du sol et production primaire (d'Aguilar et al, eds) INRA, Paris, 329-333

Zinkler D (1983) Ecophysiological adaptations of litterdwelling Collembola and tipulid larvae. In: New trends in soil biology (P Lebrun, HM Andre, A De Medts, C Gregoire-Wibo, G Wauthy, eds) DieuBrichart, Ottignies-Louvain la Neuve, 335-343 